\title{
Bone marrow granulomas: A retrospective study of 47 cases (A single center experience)
}

\author{
Sampath Kumar-Kandala Jeevan, Roshni Paul-Tara, Shantiveer Uppin, Megha Uppin \\ Departments of Pathology, Nizam's Institute of Medical Sciences, Hyderabad, India
}

Email address:

kmcsampath@gail.com (S. Kumar-Kandala J.)

\section{To cite this article:}

Sampath Kumar-Kandala Jeevan, Roshni Paul-Tara, Shantiveer Uppin, Megha Uppin. Bone Marrow Granulomas: A Retrospective Study of 47 Cases (A Single Centre Experience). American Journal of Internal Medicine. Vol. 2, No. 5, 2014, pp. 90-94. doi: 10.11648/j.ajim.20140205.13

\begin{abstract}
Background: Granulomas in bone marrow are an infrequent finding; however several diseases may be associated with granuloma formation and an etiologic diagnosis is essential. Bone marrow examination plays an important role in the diagnosis of various disorders associated with bone marrow granulomas and is useful in the investigation of pyrexia of unknown origin (PUO) as it leads to an etiological diagnosis in many of the cases. Aim: This study was undertaken to ascertain the frequency and etiological background of bone marrow granulomas. Material and methods: In the present study, forty seven cases with bone marrow granulomas were included. Clinical details, peripheral blood and marrow morphological findings were analyzed. Results: Pyrexia of unknown origin was the commonest presentation and anemia was noted in all cases. Twenty five cases had associated clinical conditions, including 7 with past history of tuberculosis and 8 with retroviral disease. Of the 47 cases, 7 showed granulomas in bone marrow aspiration, while bone marrow biopsy was diagnostic in all cases. Caseous necrosis was seen in 11(23.4\%) cases. Acid fast bacilli were demonstrated in one bone marrow aspirate. Culture studies grew Brucella organisms in one case. Tuberculosis was the commonest in the present study as compared to other studies probably due to the endemicity of tuberculosis in this region. Conclusion: If the granuloma is associated with caseous necrosis and Langhan giant cells and correlated with clinical features, a possibility of the tuberculous etiology may be suggested to allow empirical treatment to be initiated before microbiological confirmation.
\end{abstract}

Keywords: Bone Marrow Aspiration, Granuloma, PUO, Trephine Biopsy, Tuberculosis

\section{Introduction}

Bone marrow examination plays an important role in the diagnosis of haematological and non-haematological malignancies as well as various other diseases1. It is one of the commonly done procedures in the investigation of pyrexia of unknown origin (PUO) 2. Granulomas are found in 0.5 to $2.2 \%$ of trephine biopsies3-6. Diseases that are associated with bone marrow granulomas include a variety of infections, connective tissue disorders, lymphoma and metastatic carcinoma4.

The first published article on bone marrow granulomas was by Pease in 1956 . It stated that in approximately $25 \%$ of cases with bone marrow granulomas, etiology could not be detected 7. By correlating the morphological features of the granulomas with the clinical features, special stains, culture studies and newer ancillary techniques, a definite etiological diagnosis can be made in most cases. The present study was conducted to compare the role of bone marrow aspiration (BMA) cytology and bone marrow biopsy (BMB) and to formulate an effective and rapid method for diagnosing bone marrow granulomas. We also tried to study the spectrum of disorders presenting with bone marrow granulomas.

\section{Material and Methods}

All cases with bone marrow granulomas diagnosed between January 2007 to July 2010 were included in this study. The case records of these patients along with clinical details, biochemical and culture studies were analyzed. Complete blood counts were recorded and peripheral blood smear was reviewed. BMA and $\mathrm{BMB}$ were done in the same sitting under local anesthesia. Two of the representative smears were stained with Giemsa stain and the biopsy was paraffin embedded and sections stained with Haematoxylin and Eosin. The morphological features of the marrow that were studied 
included marrow cellularity, location \& morphology of the granulomas, fibrosis, caseous necrosis and inflammatory cells like plasma cells, histiocytes. All cases were stained with special stains like Ziehl-Neelsen stain for demonstration of acid fast bacilli and Methenamine Silver stain for fungi. The morphological features of granulomas were correlated with microbiology culture studies, wherever available.

\section{Results}

Of the three thousand three hundred and forty one bone marrow trephine biopsies performed at our institute over the study period, 47 cases (1.4\%) showed presence of epithelioid granulomas in the bone marrow. The age of the patients ranged from 16 to 58 years, with a median age of 35 years. The male: female ratio was $1.5: 1$. In 47 of these cases, 26 had associated clinical conditions with some form of immunosuppression (7 with past history of tuberculosis and 8 were HIV positive, 5 were diabetes mellitus, 3 had history of renal transplantation and one patient of AML, Hodgkin lymphoma and Ewing's sarcoma respectively each). Pyrexia of unknown origin was the commonest presentation seen in 47 $(100 \%)$ cases. Nineteen $(40.5 \%)$ cases had generalized weakness. Anaemia was present in all cases. Pancytopenia and bicytopenia was observed in 10 cases \& in 14 cases respectively. Blood and bone marrow culture reports for tuberculosis, typhoid and Brucella were available in 7 cases. None of the culture reports showed any significant findings except one case, which showed Brucella growth on bone marrow culture. Serological studies were done to rule out the possibility of connective tissue disorders.

\subsection{Bone Marrow Aspiration Findings}

Table 1. Bone marrow aspirations(BMA) findings

\begin{tabular}{ll}
\hline BMA findings & No of patients $(\mathbf{n}=\mathbf{4 7})$ \\
\hline Granulomas & $7(14.9 \%)$ \\
Cellularity & \\
Increased & $21(44.6 \%)$ \\
Decreased & $9(19.1 \%)$ \\
Aparticulate & $3(6.38 \%)$ \\
Normal & $14(29.78 \%)$ \\
Erythropoiesis & \\
Megaloblastoid & $3(6.4 \%)$ \\
Normoblastic & $44(93.6 \%)$ \\
Lymphoplasmacytic prominence & $29(61.7 \%)$ \\
Histiocyte prominence & $9(19.1 \%)$ \\
\hline
\end{tabular}

Bone marrow aspiration smears were hypercellular in 21 $(44.6 \%)$, normocellular in $14(29.78 \%)$, hypocellular in 9 $(19.4 \%)$ cases and in three $(6.38 \%)$ cases it was aparticulate. The aspiration cytology smears revealed epithelioid cell granulomas in $7(14.8 \%)$ cases. Erythropoiesis was normoblastic in $44(93.6 \%)$ and megaloblastoid in $3(6.38 \%)$ cases. Myeloid series and megakaryocytes were unremarkable. There was prominence of lymphocytes and plasma cells in $62 \%$ cases and histiocytic aggregates in 19\% cases. Acid fast bacilli were demonstrated in one case (Fig-1. E). Stains for fungi were negative. The details of the bone marrow aspiration findings are given in Table 1.

Table 2. Bone marrow biopsy(BMB) findings

\begin{tabular}{ll}
\hline BMB findings & No of patients $(\mathbf{n}=\mathbf{4 7})$ \\
\hline Well formed granulomas with giant cells & $26(55.3 \%)$ \\
Ill defined granulomas & $21(44.6 \%)$ \\
Caseous necrosis & $11(23.4 \%)$ \\
Location of granulomas & \\
Para-trabecular & $15(32 \%)$ \\
Interstitial & $32(68 \%)$ \\
Reed Sternberg cells & $1(2.1 \%)$ \\
Ziehl- Neelsen stain & $1(2.1 \%)$ \\
Silver methanamine & $0 \%$ \\
Reticulin - Increased condensation in \& & $10(21.2 \%)$ \\
around granulomas & \\
\hline
\end{tabular}

\subsection{Bone Marrow Biopsy Findings}

The histopathology of the trephine sections revealed 47 cases of granulomatous inflammation with granulomas ranging from one to three in number per section. They were mainly interstitial (68\%) in location with $32 \%$ being paratrabecular (Fig-1. B). Well-formed granulomas, composed of epithelioid cells surrounded by Langhan's giant cells and lymphocytes were seen in $26(55.3 \%)$ and ill-formed epithelioid granulomas in 21 (44.6\%). Caseous necrosis was noted in $11(23.4 \%)$ cases. There was increased reticulin condensation especially in and around the granulomas in 10 cases (21.2\%). Stains for Acid fast bacilli and fungi were negative in all the cases. The details of the bone marrow biopsy findings are given in Table 2. In 8 HIV positive cases, ill-defined granulomas were seen in 5 cases and the remaining cases showed well formed granulomas.

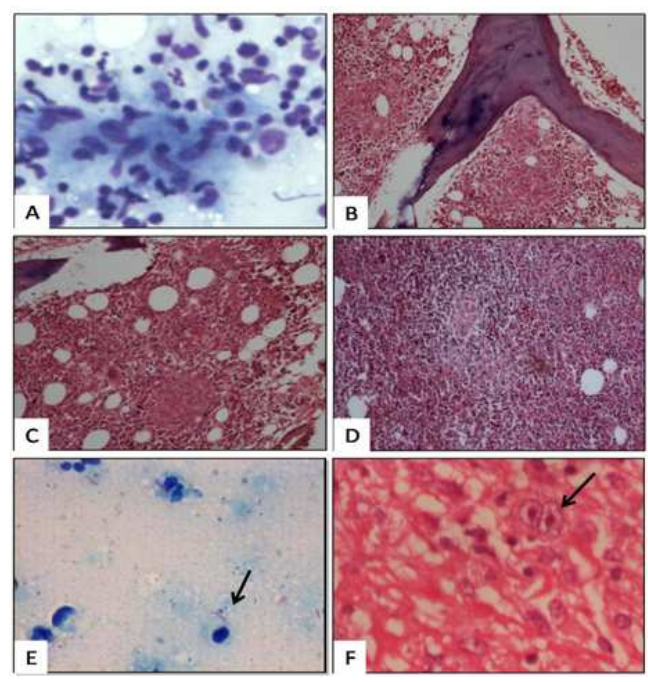

Figure 1. A) BMA - epithelioid cell aggregation (granuloma). B) BMB. Granuloma located at paratrabecular region. C) BMB - Granuloma located at interstitial region. D) BMB - Granuloma with lymphocytic cuffing. E) BMA - Ziehl- Neelsen stain: highlighting the Acid fast bacilli. F) Reed Steinberg berg cell.

Seven of the cases were empirically started on antituberculous treatment. Few cases had underlying immunosuppressive states like post-renal transplant status, 
AML and HL on treatment, Ewing's sarcoma and Diabetes mellitus which presented as pyrexia of unknown origin. In all these cases, granulomas were identified in the bone marrow. There was one case that showed few large binucleated cells with prominent nucleoli (?Reed- Sternberg cells); apart from granulomas. This was later diagnosed as Hodgkin lymphoma and proved with immunohistochemistry on lymph node biopsy.

\section{Discussion}

Granulomatous inflammation is a distinctive pattern of chronic inflammation that is encountered as a response to a persistent antigenic stimulus and characterised by aggregates of epithelioid cells, giant cells and surrounded by mononuclear cells with or without caseous necrosis.

Granulomas have been identified in a wide range of illnesses7. Morphology of granulomas in the bone marrow is similar to those seen in other organs. Granulomas in bone marrow are an uncommon finding, were detected in $1.4 \%$ of trephine biopsies in the study period, which is comparatively higher than the other studies3-6, but lower than one Indian study $-2.46 \%$ described by Basu et al 8 .

Pyrexia of unknown origin (PUO) was the commonest presentation in the present study. Patients with PUO origin were more likely to have marrow granulomas than patients biopsied for other reasons5,9. BMB and BMA are important diagnostic tools in arriving at an etiological diagnosis of patient with long-duration febrile illness, and should be routinely performed in such cases to allow treatment to be initiated before microbiological confirmation and antibiotic susceptibility 10 reports are available.

Haematological parameters were altered in all the cases. Anemia was the commonest finding in all patients. Pancytopenia was seen in $10(21.3 \%)$ and bicytopenia in $14(29.7 \%)$ cases. Pancytopenia, anemia, leukopenia and thrombocytopenia have been reported in patients with granulomatous bone marrow disease 4 .

Granulomas in marrow aspiration cytology smears are difficult to identify probably because of the fibrosis in and around the granulomas. In the present study, granulomas were detected in $7 / 47$ (14.8\%) of marrow aspirates (Fig-1. A), which is comparatively higher than that detected by Basu et al8 (1/14 cases, $7.14 \%)$ and Chandra et al11 (0/6 cases, $0 \%)$. All these 7 cases were subsequently confirmed on marrow biopsy sections and $100 \%$ positive correlation was found between bone marrow aspiration and biopsy. BMB is a better tool than BMA to demonstrate granulomas as the morphology is better preserved and more amount of tissue is available for study. The detection rates are higher if serial sections of trephine biopsies are studied when the original sections do not show granulomas in suspected cases 12 .

Seven of the cases who were already on anti-tuberculosis treatment had undergone bone marrow aspiration and biopsy due to persistent fever not responding to therapy. Acid fast bacilli were detected in one case by Ziehl-Neelsen stain. These were considered as disseminated tuberculosis owing to clinical details and characteristic morphology. Tuberculosis is one of the most frequent causes of bone marrow granuloma constituting $6 \%$ to $48 \%$ of the cases 13 .

There were $8 \mathrm{HIV}$ positive cases presenting with PUO which had granulomas in the bone marrow. Of the 8 cases, 5 cases had pulmonary tuberculosis. Acid fast bacilli and fungi could not be identified in both BMA and BMB. Culture studies were also not helpful in these cases. As expected, there were more number of cases with ill-defined granulomas $(5$ cases, 62.5\%) than well-defined granulomas (3 cases, 37.5\%). Bone marrow abnormalities are seen in $90 \%$ of HIV infected cases during the course of the disease in form of increased cellularity, dyspoiesis and granulomatous inflammation 14. Exact mechanism of HIV induced marrow changes are not known, however there are possibly due to either direct effect of HIV or opportunistic infections or by anti-retroviral drugs 15.

Malignant neoplasms have been reported as the cause of bone marrow granulomas in $20 \%$ to $25 \% 3-5,13$. Acute myeloid leukemia (AML) is an aggressive hematolymphoid neoplasm with characteristic bone marrow findings. Some studies have led to a hypothesis for granulomas in bone marrow that there is a strong induction of anti-leukemic immune response to tumor-associated antigens and a high incidence of circulating immune complexes in AML patients16, 17. In this study, there was a case of treated AML, with a granuloma in bone marrow. Regarding the association between AML and granulomas - it is not sure whether the relationship is causal or coincidental. There was one case with few large binucleated cells and prominent nucleoli in addition to the granuloma in bone marrow (Fig-1.F). This was later proved as Hodgkin lymphoma on lymph node biopsy. The presence of a granulomatous lesion along with a hematolymphoid neoplasm has been associated with a good prognosis; particularly in patients with Hodgkin lymphoma18, 19.

There were three post-renal transplant patients presenting with PUO, showing marrow granulomas. These transplant patients are immunosuppressed and are prone to develop various types of opportunistic infections including TB, and a majority of these infections are a result of reactivation of dormant disease. The prevalence of TB among renal transplant recipients varies widely in different parts of the world and ranges from less than $1 \%$ in Western countries and nearly $15 \%$ in India20-24. A single follow up case of Ewing's sarcoma and five cases of diabetes mellitus, presenting with PUO, showed granulomas in bone marrow. This can be explained by immunosuppressed state of the patients.

Bone marrow cultures (BMCs) and blood cultures (BCs) are frequently obtain in the evaluation of PUO and important investigations in patients with PUO. Bone marrow can be collected at same time of BMA procedure for culture studies and is especially helpful in disseminated bacterial, fungal and tuberculous infection. BMCs are particularly useful for patients who are immunocompromised. BMCs can be positive when blood culture (BC) is negative25. The viable organism counts in the bone marrow are considerably less affected by antibiotic treatment than blood counts 26 . In this study, BMC 
studies could not pick up any organisms barring one patient, where Brucella organisms were grown.

There are no distinguishing features in the morphology of bone marrow granuloma to permit a definitive diagnosis of the underlying conditions 4,7 . Though the granulomas associated with caseous necrosis and Langhan type giant cells would be seen more frequently in tuberculosis, the diagnosis cannot be made solely on pathologic criteria. Rather, it will have to be correlated with clinical, laboratory, and radiological data. In this study, the underlying illness, associated with bone marrow granulomas was identified in $36 \%$, which is much less than other studies $3,4,8$ (table-3). In the present study, infectious etiology is the commonest cause for bone marrow granulomas, similar to other studies7,8. The higher incidence of tuberculosis in the present study is due to the endemicity of tuberculosis in our country.

Table 3. Comparison of the present study with various other studies

\begin{tabular}{|c|c|c|c|c|}
\hline Feature & Bodem et al $^{4}$ & 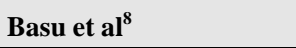 & Vilalta-Castel E et al $^{3}$ & Our study \\
\hline No. of cases studied & 58 & 14 & 40 & 47 \\
\hline Granulomas in BMA & - & $1(7.14 \%)$ & - & $7(14.9 \%)$ \\
\hline Underling etiology Identified & $87 \%$ & $78 \%$ & $82.5 \%$ & $36 \%$ \\
\hline Commonest etiology & $\begin{array}{l}\text { Infections (Rocky Mountain } \\
\text { spotted fever) }\end{array}$ & Infections (Tuberculosis) & $\begin{array}{l}\text { Infections (Tuberculosis, } \\
\text { Brucellosis) }\end{array}$ & Infections (Tuberculosis) \\
\hline
\end{tabular}

\section{Conclusion}

Bone marrow granulomas are uncommon. When detected, it is essential to look for the underlying etiology. There are no morphological features which allow reliable differentiation between the causes of granulomas, hence clinical features; morphology, culture and serology are to be combined to arrive at an etiologic diagnosis. If the granuloma is associated with caseous necrosis and Langhan giant cells and correlated with clinical features, a possibility of the tuberculous etiology may be suggested to allow empirical treatment to be initiated before microbiological confirmation. Bone marrow examination is a useful tool to diagnose possible etiology in patients with PUO. BMB is always superior to the BMA to diagnose granulomas.

\section{References}

[1] Toi PC, Varghese RG, Rai R. Comparative evaluation of simultaneous bone marrow aspiration and bone marrow biopsy: an institutional experience. Indian J. Hematol. Blood Transfus. Off. J. Indian Soc. Hematol. Blood Transfus. 2010 Jun;26(2):41-4.

[2] Laing RBS. Fever and Pyrexia of Unknown Origin. Medicine 2001; 29:24-6.

[3] Vilalta-Castel E, Valdés-Sanchez MD, Guerra-Vales JM, Teno-Esteban C, Garzón A, López JI, et al. Significance of granulomas in bone marrow: a study of 40 cases. Eur. J. Haematol. 1988 Jul;41(1):12-6.

[4] Bodem CR, Hamory BH, Taylor HM, Kleopfer L. Granulomatous bone marrow disease. A review of the literature and clinicopathologic analysis of 58 cases. Medicine (Baltimore). 1983 Nov;62(6):372-83.

[5] Bhargava V, Farhi DC. Bone marrow granulomas: clinicopathologic findings in 72 cases and review of the literature. Hematol. Pathol. 1988;2(1):43-50.

[6] Vijnovich Barón IA, Barazzutti L, Tartas N, Korin J, Sánchez Avalos JC. [Bone marrow granulomas]. Sangre (Barc.). 1994 Feb;39(1):35-8.
[7] PEASE GL. Granulomatous lesions in bone marrow. Blood. 1956 Aug;11(8):720-34.

[8] Basu D, Saravana R, Purushotham B, Ghotekar LH. Granulomas in bone marrow--a study of fourteen cases. Indian J. Pathol. Microbiol. 2005 Jan;48(1):13-6.

[9] Nichols L, Florentine B, Lewis W, Sattler F, Rarick MU, Brynes RK. Bone marrow examination for the diagnosis of mycobacterial and fungal infections in the acquired immunodeficiency syndrome. Arch. Pathol. Lab. Med. 1991 Nov;115(11):1125-32.

[10] Gupta R, Setia N, Arora P, Singh S, Singh T. Hematological profile in pyrexia of unknown origin: role of bone marrow trephine biopsy vis-à-vis aspiration. Hematol. Amst. Neth. 2008 Oct;13(5):307-12.

[11] Chandra S, Chandra H. Comparison of bone marrow aspirate cytology, touch imprint cytology and trephine biopsy for bone marrow evaluation. Hematol. Reports. 2011 Oct 19;3(3):e22.

[12] Nakajima M, Niki Y, Manabe T, Matsushima T. [Detection of lesions in bone marrow for the diagnosis of miliary tuberculosis: reliability of bone marrow aspiration and biopsy in view of distribution pattern of lesions in autopsy cases]. Kansenshōgaku Zasshi J. Jpn. Assoc. Infect. Dis. 1996 Sep;70(9):963-9.

[13] Eid A, Carion W, Nystrom JS. Differential diagnoses of bone marrow granuloma. West. J. Med. 1996 Jun;164(6):510-5.

[14] Paradela A, Rivas C, Fernández-Guerrero M, Román A. [Histopathology of bone marrow biopsy in patients with human immunodeficiency virus infection]. Rev. Clínica Española. 1996 Jan;196(1):9-15.

[15] Richman DD, Fischl MA, Grieco MH, Gottlieb MS, Volberding PA, Laskin OL, et al. The toxicity of azidothymidine (AZT) in the treatment of patients with AIDS and AIDS-related complex. A double-blind, placebo-controlled trial. N. Engl. J. Med. 1987 Jul 23;317(4):192-7.

[16] Greiner J, Schmitt M, Li L, Giannopoulos K, Bosch K, Schmitt A, et al. Expression of tumor-associated antigens in acute myeloid leukemia: Implications for specific immunotherapeutic approaches. Blood. 2006 Dec 15;108(13):4109-17. 
[17] Kishore J, Kumar R, Choudhry VP, Bhargava M. Circulating immune complexes in leukaemias and lymphomas. Indian J. Pediatr. 1992 Apr;59(2):225-31.

[18] Sacks EL, Donaldson SS, Gordon J, Dorfman RF. Epithelioid granulomas associated with Hodgkin's disease: clinical correlations in 55 previously untreated patients. Cancer. 1978 Feb;41(2):562-7.

[19] Abrams J, Pearl P, Moody M, Schimpff SC. Epithelioid granulomas revisited: long-term follow-up in Hodgkin's disease. Am. J. Clin. Oncol. 1988 Aug;11(4):456-60.

[20] Lui SL, Tang S, Li FK, Choy BY, Chan TM, Lo WK, et al. Tuberculous infection in southern Chinese renal transplant recipients. Clin. Transplant. 2004 Dec;18(6):666-71.

[21] Wang B, Lü Y, Yú L, Liu C, Wu Z, Pan C. Diagnosis and treatment for tuberculosis infection in liver transplant recipients: case reports. Transplant. Proc. 2007 Dec;39(10):3509-11.
[22] Higgins RM, Cahn AP, Porter D, Richardson AJ, Mitchell RG, Hopkin JM, et al. Mycobacterial infections after renal transplantation. Q. J. Med. 1991 Feb;78(286):145-53.

[23] Queipo JA, Broseta E, Santos M, Sánchez-Plumed J, Budía A, Jiménez-Cruz F. Mycobacterial infection in a series of 1261 renal transplant recipients. Clin. Microbiol. Infect. Off. Publ. Eur. Soc. Clin. Microbiol. Infect. Dis. 2003 Jun;9(6):518-25.

[24] Prasoon D. Acid-fast bacilli in fine needle aspiration smears from tuberculous lymph nodes. Where to look for them. Acta Cytol. 2000 Jun;44(3):297-300.

[25] Jha A, Sarda R, Gupta A, Talwar OP. Bone marrow culture vs. blood culture in FUO. Jnma J. Nepal Med. Assoc. 2009 Jun;48(174):135-8.

[26] Wain J, Pham VB, Ha V, Nguyen NM, To SD, Walsh AL, et al. Quantitation of bacteria in bone marrow from patients with typhoid fever: relationship between counts and clinical features. J. Clin. Microbiol. 2001 Apr;39(4):1571-6. 